\title{
API2CAN: a dataset \& service for canonical utterance generation for REST APIs
}

\author{
Mohammad-Ali Yaghoub-Zadeh-Fard ${ }^{*}$ and Boualem Benatallah
}

\begin{abstract}
Objectives: Recently natural language interfaces (e.g., chatbots) have gained enormous attention. Such interfaces execute underlying application programming interfaces (APIs) based on the user's utterances to perform tasks (e.g., reporting weather). Supervised approaches for building such interfaces rely upon a large set of user utterances paired with APIs. Collecting such pairs is typically starts with obtaining initial utterances for a given API method. Generating initial utterances can be considered as a machine translation task in which an API method is translated into an utterance. However, the key challenge is the lack of training samples for training domain-independent translation models. In this paper, we propose a dataset for training supervised models to generate initial utterances for APIs.

Data description: The dataset contains 14,370 pairs of API methods and utterances. It is built automatically by converting method descriptions of a large number of APIs to user utterances; and it is cleaned manually to ensure quality. The dataset is also accompanied with a set of microservices (e.g., translating API methods to utterances) which can facilitate the process of collecting training samples for building natural language interfaces.
\end{abstract}

Keywords: Chatbots, Bot development, Natural language interfaces

\section{Objective}

Recently natural language interfaces (also known as bots, chatbots, dialog systems, and virtual assistants) have attracted enormous attention because of their potentials in hands-free environments (e.g., self-driving cars) and accessibility products [1]. Building such interfaces often requires training utterances (e.g., book a flight from Sydney to Paris), annotated with user intents (e.g., bookflight) and associated parameters ${ }^{1}$ (e.g., from = "Sydney", to $=$ "Paris") $[1,2]$. Training utterances are used to train supervised models for detecting the users' intent based on their utterances. Because of the richness of human language, collecting large and diverse set of annotated utterances is required for building efficient natural language interfaces [2]. This is typically done in two steps: (i) generating an initial utterance (known as canonical utterance), and (ii) paraphrasing it to obtain more diverse

*Correspondence: m.yaghoubzadehfard@unsw.edu.au UNSW Sydney, Kensington, Australia training utterances [3-5]. In this paper, we focus on the first step, particularly for REST (Representational State Transfer) APIs since they are one of the most common forms of intents [6].

With the growing number of REST APIs and their everchanging interfaces (e.g., renaming parameters, adding/ removing operations), virtual assistants now require to automatically generate canonical utterances for scalability $[4,5]$. Research has shown the feasibility of leveraging supervised machine translation techniques to generate canonical utterances for REST APIs [6, 7]. Ironically, such machine translation approaches also require training datasets to learn how to map REST API operations to canonical utterances. In this paper, we introduce the API2CAN dataset containing a large set of REST API operations (e.g., GET/series/\{id\}/actors) paired with their corresponding canonical templates (a canonical utterance in which parameter values have been replaced with placeholders e.g., "get the list of actors of the TV series with id being $<i d>$ ").

1 also known as entities and slots.

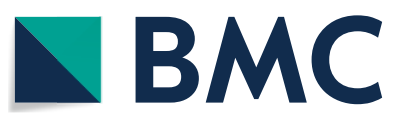

(c) The Author(s) 2021. Open Access This article is licensed under a Creative Commons Attribution 4.0 International License, which permits use, sharing, adaptation, distribution and reproduction in any medium or format, as long as you give appropriate credit to the original author(s) and the source, provide a link to the Creative Commons licence, and indicate if changes were made. The images or other third party material in this article are included in the article's Creative Commons licence, unless indicated otherwise in a credit line to the material. If material is not included in the article's Creative Commons licence and your intended use is not permitted by statutory regulation or exceeds the permitted use, you will need to obtain permission directly from the copyright holder. To view a copy of this licence, visit http://creativecommons.org/licenses/by/4.0/. The Creative Commons Public Domain Dedication waiver (http://creativeco mmons.org/publicdomain/zero/1.0/) applies to the data made available in this article, unless otherwise stated in a credit line to the data. 
Table 1 Overview of data files/data sets

\begin{tabular}{|c|c|c|c|}
\hline Label & Name of data file/data set & $\begin{array}{l}\text { File types } \\
\text { (file extension) }\end{array}$ & $\begin{array}{l}\text { Data repository and identifier (DOI or } \\
\text { accession number) }\end{array}$ \\
\hline Dataset 1 & $\begin{array}{l}\text { API2Can-train.json contains 13,029 canonical } \\
\text { utterances for } 858 \text { APIs }\end{array}$ & JavaScript Object Notation (.json) & https://doi.org/10.6084/m9.figshare.13331564 [9] \\
\hline Dataset 2 & $\begin{array}{l}\text { API2Can-validation.json contains } 433 \text { canonical } \\
\text { utterances for } 50 \mathrm{APIs}\end{array}$ & JavaScript Object Notation (.json) & https://doi.org/10.6084/m9.figshare.13332029 [10] \\
\hline Dataset 3 & $\begin{array}{l}\text { API2Can-test.json contains } 908 \text { canonical utter } \\
\text { ances for } 50 \mathrm{APIs}\end{array}$ & JavaScript Object Notation (.json) & https://doi.org/10.6084/m9.figshare.13331507 [11] \\
\hline Data file 1 & $\begin{array}{l}\text { API2Can-Schema shows a sample JSON object } \\
\text { representing a training sample in the datasets }\end{array}$ & Figure (.png) & https://doi.org/10.6084/m9.figshare.13332347 [12] \\
\hline Data file 2 & $\begin{array}{l}\text { API2Can Service contain the python scripts for } \\
\text { generating the datasets }\end{array}$ & a zip file containing python codes (.py) & https://doi.org/10.6084/m9.figshare.13332125 [13] \\
\hline
\end{tabular}

\section{Data description}

To generate the "API 2 CAN dataset", we obtained OpenAPI specifications indexed in OpenAPI Directory [8]. OpenAPI specification is a standard documentation format for documenting the interface of a REST API, including its operations and their parameters. OpenAPI Directory is a Wikipedia for REST APIs and maintains OpenAPI specifications for a large number of REST APIs. We obtained the latest version of each API index in OpenAPI Directory, and totally collected 983 APIs, containing 18,277 operations in total. Finally, we generated canonical utterances for each of the extracted operations as explained in [6]. In short, we converted the summary or description (e.g., "...gets the [actor](\\#/definitions/ Actor) by id. ...") of operations (e.g., GET /actors/(id\}) in three steps: (i) extracting a sentence starting with a verb (e.g., "gets the actor by id."), (ii) converting the extracted sentence to an imperative form to (e.g., "get the actor by id."), and (iii) injecting the parameters (e.g., "get the actor by id being<id>.") Finally, we manually cleaned the automatically generated utterances to ensure quality of the generated canonical utterances. As such we generated the API2CAN dataset which includes 14,370 pairs of operations and their corresponding canonical utterances, ignoring operations without generated canonical utterances. Next, we randomly divided the generated dataset into three parts as summarized in Table 1.

The API2CAN dataset is now public and accessible from [14]. The dataset is stored in a JSON (JavaScript Object Notation) array in which each element represents single Operation, including its API, and API version, endpoint (URL), HTTP verb (e.g., GET, POST), parameters, canonical utterances as shown in Fig. 1.

The generated canonical utterances can be used for training bots for existing REST APIs in the dataset. The API2CAN dataset can be also used to train machine translation systems to generate canonical utterances, which is required for new APIs. ${ }^{2}$ The API2CAN dataset is also accompanied with a set of microservices called API2CAN Service. The service is implemented as a standalone open-source REST Service in Python, and it is also accessible from [13]. This REST service provides several functionaries as follow:

- Parsing OpenAPI Specification This microservice parses the given API specification (in YAML format) and extract API elements such as operations and their parameters in JSON format.

- Generating Canonical Utterances This microservice generates the canonical utterances based on the approaches used in generation of the API2CAN dataset. In a nutshell, two approaches are used for generating canonical utterances as introduced in [6]: (i)

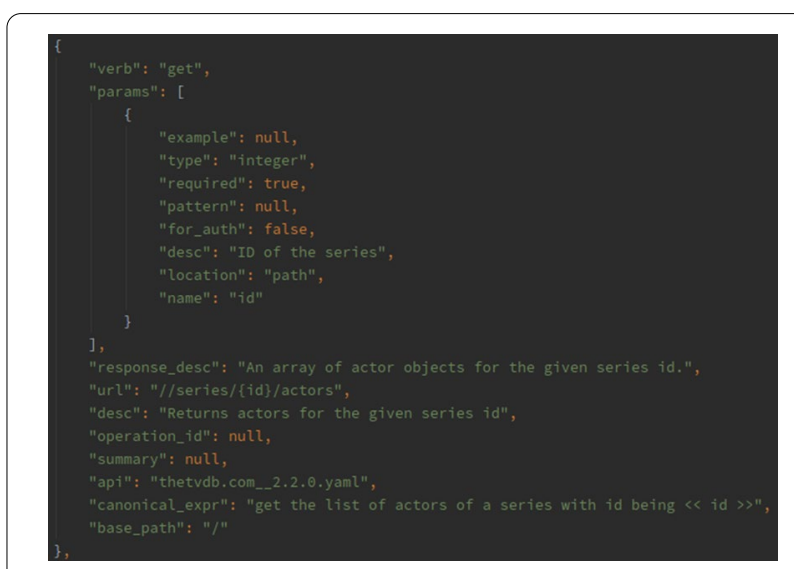

Fig. 1 Dataset schema-sample operation [https://doi.org/10.6084/ m9.figshare.13332347]

\footnotetext{
${ }^{2}$ APIs which are not included in the proposed dataset.
} 
converting operation summary as briefly introduced in the previous section, and (ii) the resourced-based translator which relays on the notion of Resources in REST APIs and is proposed in $[6,7]$.

- Sampling Parameter Values This microservice generates values (e.g., "Sydney", "Paris") for the parameters (e.g., "to") of the given operation based on the approaches introduced in [6]. Generated values can be used to populate placeholders inside generated canonical utterances (e.g., "book a flight to Sydney").

\section{Limitations}

Given that fulfilling complex intents usually requires a combination of operations $[4,15]$, it is also needed to generate canonical utterances for compositions between operations. To achieve this, it is required to detect the relations between operations and generate canonical templates for complex tasks (e.g., tasks requiring conditional operations or compositions of multiple operations). Adding such cases thus require further research.

\section{Abbreviations}

API: Application Programming Interfaces; REST: Representational State Transfer; JSON: JavaScript Object Notation; URL: Uniform Resource Locator; HTTP: Hypertext Transfer Protocol.

\section{Acknowledgements}

This research was supported by the Australian Government.

\section{Authors' contributions}

The authors of this article, MY and BB, contributed to the conceptualization of the work. Both authors read and approved the final manuscript.

\section{Funding}

This research was supported fully by the Australian Government through the Australian Research Council's Discovery Projects funding scheme (project DP1601104515). This research is also supported by the Australia's Government by "Australian Government Research Training Program Scholarship".

\section{Availability of data and materials}

The data described in this Data note can be freely and openly accessed on Figshare[9-14]. Please see Table 1 and reference list for details and links to the data.

\section{Declarations}

Ethics approval and consent to participate Not applicable.

\section{Consent for publication}

Not applicable.

\section{Competing interests}

The authors declare that they have no competing interests.

Received: 8 December 2020 Accepted: 5 May 2021

Published online: 22 September 2021

\section{References}

1. Yaghoub-Zadeh-Fard M, Benatallah B, Casati F, Chai Barukh M, Zamanirad S. User utterance acquisitionfor training task-oriented bots: a review of challenges, techniques and opportunities. IEEE Internet Comput. 2020. https://doi.org/10.1109/MIC.2020.29781572.

2. Campagna G, Ramesh R, Xu S, Fischer M, Lam MS. Almond: the architecture of an open, crowdsourced, privacy-preserving, programmable virtual assistant. In: Proceedings of the 26th International Conference on World Wide Web. WWW '17. International World Wide Web Conferences SteeringCommittee, Republic and Canton of Geneva, Switzerland; 2017, pp. 341-50. doi:https://doi.org/10.1145/3038912.3052562. https://doi. org/10.1145/3038912.30525623.

3. Yaghoub-Zadeh-Fard M, Benatallah B, Casati F, Barukh MC, Zamanirad S. Dynamic wordrecommendation to obtain diverse crowdsourced paraphrases of user utterances. In: Patern 'o F, Oliver N, Conati C, Spano LD, Tintarev N (eds.) IUI '20: 25th International Conference on Intelligent User Interfaces, Cagliari, Italy, March 17-20, 2020, pp. 55-66. ACM, 2020. doi: https://doi.org/10.1145/3377325.3377486. https://doi.org/10.1145/33773 25.33774864 .

4. Negri M, Mehdad Y, Marchetti A, Giampiccolo D, Bentivogli L. Chinese whispers: cooperative paraphraseacquisition. In: Proceedings of the Eighth International Conference on Language Resources and Evaluation(LREC'12), pp. 2659-65. European Language Resources Association (ELRA), Istanbul, Turkey; 2012.http://www.Irec-conf.org/proce edings/lrec2012/pdf/772Paper.pdf5.

5. Su Y, Awadallah AH, Khabsa M, Pantel P, Gamon M, Encarnacion M. Building natural languageinterfaces to web apis. In: Proceedings of the 2017 ACM on conference on information and knowledge management. CIKM '17. ACM, New York, NY, USA; 2017, pp. 177-86. doi:https://doi.org/ 10.1145/3132847.3133009. http://doi.acm.org/https://doi.org/10.1145/ 3132847.31330096

6. Yaghoub-Zadeh-Fard M, Benatallah B, Zamanirad S. Automatic canonical utterance generation fortask-oriented bots from API specifications. In: Bonifati A, Zhou Y, Salles MAV, B'ohm A, Olteanu D, Fletcher GHL, Khan $A$, Yang B (eds.) Proceedings of the 23nd international conference on extending database technology, EDBT 2020, Copenhagen, Denmark, March 30-April 02, 2020. OpenProceedings.org, 2020, pp. 1-12. doi:https://doi.org/10.5441/002/edbt.2020.02. https://doi.org/https://doi. org/10.5441/002/edbt.2020.027.

7. Yaghoub-Zadeh-Fard M-A, Zamanirad S, Benatallah B. Rest2bot: bridging the gap between botplatformsand rest apis. In: The Web Conference; 2020, p. 8.

8. Goncharov I, Dubovyk S, Hotsi R. APIs-guru. https://github.com/APIsguru/openapi-directory/tree/master/APIs

9. Yaghoub-Zadeh-Fard M, Benatallah B. API2Can-train. figshare. Dataset; 2020. https://doi.org/10.6084/m9.figshare.13331564

10. Yaghoub-Zadeh-Fard M, Benatallah B. API2Can-validation. figshare. Dataset; 2020. https://doi.org/10.6084/m9.figshare.13332029

11. Yaghoub-Zadeh-Fard M, Benatallah B. API2Can-test. figshare. Dataset; 2020. https://doi.org/10.6084/m9.figshare.13331507

12. Yaghoub-Zadeh-Fard M, Benatallah B. API2CAN Schema. figshare. Figure; 2020. https://doi.org/10.6084/m9.figshare.13332347

13. Yaghoub-Zadeh-Fard M, Benatallah B. API2CAN Service. figshare. Software; 2020. https://doi.org/10.6084/m9.figshare.13332125

14. Yaghoub-Zadeh-Fard M, Benatallah B. API2CAN. figshare. Collection; 2021. https://doi.org/10.6084/m9.figshare.c.5404539

15. Fast $\mathrm{E}$, Chen B, Mendelsohn J, Bassen J, Bernstein MS. Iris: a conversational agent for complex tasks. In: Proceedings of the $2018 \mathrm{CHI}$ conference on human factors in computing systems. CHI '18, pp.473-147312. ACM, New York, NY, USA; 2018. doi:https://doi.org/10.1145/3173574.3174047. http:// doi.acm.org/https://doi.org/10.1145/3173574.3174047

\section{Publisher's Note}

Springer Nature remains neutral with regard to jurisdictional claims in published maps and institutional affiliations. 